Golduoppelsalz: $\mathrm{C}_{7} \mathrm{H}_{7}\left(\mathrm{C}_{7} \mathrm{H}_{7}\right) \mathrm{N}_{1} \mathrm{O}_{2}, \mathrm{HCl}+\mathrm{AuCl}_{8}$. Gelbe, seidenglänzende, bei $104^{\circ}$ schmelzende, in Wasser schwer loslich $\theta$ Krystallnadeln, welche sich nur aus verdunntem Alkohol, bei Gegenwart ron etwas Goldchlorid, umkrystallisieren lassen.

$0,2637 \mathrm{~g}$ enthielten 0,0852 g Au.

Gefunden: $\quad$ Berechnet făc $\mathrm{C}_{7} \mathrm{H}_{7}\left(\mathrm{C}_{7} \mathrm{H}_{7}\right) \mathrm{N}_{4} \mathrm{O}_{2}, \mathrm{HCl}+\mathrm{AaC}_{\mathrm{a}}$ :

An 38,30 32,26 .

Platindoppelsalz: $\quad\left[\mathrm{C}_{7} \mathrm{H}_{7}\left(\mathrm{C}_{7} \mathrm{H}_{7}\right) \mathrm{N}_{4} \mathrm{O}_{9}, \mathrm{HCl}\right]_{2} \mathrm{PtCl}_{4}$. Das Platindoppelsalz des Benzyl Theophyllins ist in Wasser leichter loslich als das entsprechende Golddoppelsalz. Es scheidet sich beim Verdunsten seiner wässerigen, Platinchlorid im Ueberschaß enthaltenden Lösung in krystallwasserfreien, rotgelben, kompakten Nadeln aus.

$0,3663 \mathrm{~g}$ enthielten $0,0754 \mathrm{~g} \mathrm{Pt}$.

Gefunden: Berechnet für $\left[\mathrm{C}_{7} \mathrm{H}_{7}\left(\mathrm{C}_{7} \mathrm{H}_{2}\right) \mathrm{N}_{4} \mathrm{O}_{2}, \mathrm{HCl}\right]_{8} \mathrm{Pt} \mathrm{Cl}$ :

Pr 20,59 20,69 .

\title{
Untersuchung der Frucht von Styrax Obassia Siebold et Zuccarini.
}

Von Y. Asahina,

Assistent an dem pharmazeutischen Institut der medizinischen Fakultat der Kaiserlichen Universität Tokyo (Japan).

(Eingegangen den 18. VI. 1907.)

Es gibt in Japan zwei Styraxarten, nämlich Styrax japonica Siebold et Zuccarini and Styrax Obassia Siebold et Zuccarini. Die Fruchtschalen der ersteren benutzt man in Japan als Fischfangmittel, indem man sie mit Sand zerst8Bt und in den Fluß oder in den Teich hinein wirft.

S. Keïmatsu ${ }^{1}$ ) hat die Bestandteile der Fruchtschalen von $S$. japonica untersucht und darin ein krystallisiertes, aut Fische giftig wirkendes Saponin (Styrasaponin genannt) von der Zusammensetzung $\mathrm{C}_{88} \mathrm{H}_{60} \mathrm{O}_{18}$, gefanden.

Da man bisher nichts Bemerkenswertes uber die physiologische Wirkung der Frucht von Styrax Obassia kennt, so schien es mir

1) S. Keimatsa. Journal of the Tokyo cbemical society, Bd. 25, Ne. 11 (japanisch). 
interessant, dieselbe elner ohemischen Studie za unterziehen und die Bestandteile beider Pflanzen zu vergleichen.

Die im Angust gesammelten reifen Früchte von S. Obassia wurden einige Tage an der Sonne getrocknet. Die von Kelchen und Samen getrennten Fruchtschalen wurden mit $60 \%$ igem Weingeist ausgekocht, der Auszug wurde hieraut durch Destillation von Alkohol befreit und der Rtickstand schlieblich auf dem Wasserbade zur Sirnpkonsistenz eingedampft. Das Extrakt wurde alsdann mit Magnesia usta gemischt und eingetrocknet. Die so erhaltene hygroskopische Masse warde mit 80\%igem Alkohol ausgezogen. Beim Einengen der alkoholischen Lrsung bis zum 1/8 Volum, trat eine reichliche Krystallabscheidung ein. Auch aus der Mutterlange wurden durch Verdampfen bis zar Sirupkonsistenz und Behandeln des Rückstandes mit starkem Alkohol noch reichliche Mengen derselben Krystalle erhalten. $2 \mathrm{~kg}$ lufttrockener Fruchtschalen ergaben etwa $200 \mathrm{~g}$ Rohkrystalle: Ausbeute $10 \%$. Die Rohkrystalle waren schon ziemlich wei, nur beim Anflosen in Wasser trat Trtbung ein. Zur Reinigung worden die Krystalle in Wasser gelöst, das klare Filtrat zum Sirup verdampft und der Rückstand aus 95\%igem Alkohol umkrystallisiert.

Die reinen Krystalle stellen schneeweiße Prismen vom Schmp. $155^{\circ}$ dar. Sie schmecken anfangs staßlich, dann bitterlich und sind ziemlich hygroskopisch. Diese Krystalle sind sehr leicht loslich in Wasser; die Lösung reagiert neutral. In kaltem starkem Alkohol ist diese Verbindung schwer löslich, in Aether, Benzol und Aceton fast unlöslich. In konzentrierter Schwetelsăure löst sie sich in der Kälte ohne Färbung, beim Erwărmen wird die Lơsung braun getărbt. Konzentrierte Salpetersăure nimmt diese Krystalle leicht auf; Wasserzusatz ruft in der Lðsung keine Füllung hervor. Die Krystalle enthalten keinen Stickstoff. Die wässerige Lösung derselben reduziert die Fehling'sche Lð̋sng, selbst nach dem Erhitzen mit verdünnten Mineralsäuren, nicht. Ammoniakalische Silberlosung wird in der Wärme reduziert unter Spiegelbildnng. Die Krystalle verbinden sich in wăsseriger Lösnog nicht mit Phenylhydrazinacetat. Auch die Molisch'sche Znckerreaktion ergab nur ein negatives Resultat. Durch Oxydation mit unterbromigsanrem Natrinm oder mit Salpetersäure wird jedoch eine, die Fehlin g'sche Lösung reduzierende Flìssigkeit erbalten. Die wăsserige Lð̋sung der Krystalle wird weder durch Kupfersulfatlösung, welche möglichst wenig Ammoniak enthk̈lt, noch durch Bleizucker oder Bleiessig gefallt, wohl aber durch Bleiessig und Ammoniak. Die wăsserige Lơsung ist linksdrehend, and $z$ war $[a]_{D}^{20}=-71,72^{\circ}$. Die wässerige Losang der Krystalle wird durch Saccharomyces Pasteurianus, nicht dagegen durch Champagnerhefe und Saccharomyces anomalus in Gärnng versetzt. 
Die Elementaranalysen der bei $100^{\circ}$ getrockneten Substanz ergaben folgende Resultate:

1. $0,3890 \mathrm{~g}$ lieferten $0,2600 \mathrm{~g} \mathrm{H}_{2} \mathrm{O}$ and $0,6240 \mathrm{~g} \mathrm{CO}$.

2. $0,2755 n$ " 0,1850 n $n 0,4406$ n n

3. $0,2144 n$ n $0,1487 n$ n 0,3438 n

\begin{tabular}{|c|c|c|c|}
\hline Berechnet far & & fande & \\
\hline $\mathrm{C}_{6} \mathrm{H}_{18} \mathrm{O}_{5}$ : & 1. & 2. & 3. \\
\hline C $\quad 48,90$ & 43,77 & 43,59 & 43,73 \\
\hline Н $\mathbf{7 , 3 1}$ & 7,49 & 7,51 & 7,79 . \\
\hline
\end{tabular}

Unter den Verbindungen, welche die Zusammensetzang $\mathrm{C}_{6} \mathrm{H}_{12} \mathrm{O}_{5}$ besitzen, steht der Schmelzpunkt des $\beta$-Methylglykosids dem der untersuchten Krystalle sehr nahe. $\mathrm{Da}$ aber meine Krystalle kein Glykosid sind, so ist die Identitat beider Snbstanzen ausgeschlossen. Ich schlage vor, die Krystalle Styracit za nennen.

\section{Elmwirkung von Essigsåureanhydrid auf Styraolt.}

$5 \mathrm{~g}$ Styracit wurden mit $5 \mathrm{~g}$ entwässertem Natriumacetat und $80 \mathrm{ccm}$ Essiggäureanhydrid in einem mit Kühlrohr versehenen Kölbchen versetzt und in einem Paraffinbade 2 Stunden in gelindem Sieden gehalten. Nach dem Erkalten wurde das Reaktionsprodakt mit Wasser versetzt, wobel kein Niederschlag entstand. Die Lösang wurde alsdann auf dem Wasserbade verdampft, um den Ueberschal an Essigsäure zu verjagen. Der sirupartige Ruckstand warde hieranf mit Aether geschilttelt. Beim Yerdampfen des Aethers blieb jedoch nichts zurtick.

Der Acetylierangsversuch wurde unter Zusatz von Zinkchlorid ernevert, doch konnte ich außer Haminsubstanz nichts Faßbares erhalten.

\section{Einwirkung von Benzoyichlorid auf Styraolt.}

$5 \mathrm{~g}$ Styracit warden in $10 \%$ iger Natronlange gelöat und mit $10 \mathrm{~g}$ Benzoylchlorid stark geschüttelt. Hierbei wurde eine weiße, klebrige Substanz niedergeschlagen. Dieselbe ist in Aether unlöslich; wegen der klebrigen Beschaffenheit konnte ich jedoch diese Substanz nicht analysenrein erbalten.

Die Benzoylierung in Pyridinlosung ergab ebenfalls nar eine sirupartige, in Wasser unlösliche Substanz.

\section{Elnwirkung von Benzaldehyd auf Styracit.}

Zur Prüfung, ob Styracit eine Benzalverbindung wie Mannit, Dulcit u. a. liefert, warde er mit 50\% iger Schwefelsänre und Benzaldehyd geschuttelt. Selbst nach wochenlangem Stehen konnte jedoch kein Kondensationsprodukt nachgewiesen werden. 


\section{Einwirkung von Jodwasserstoffisăure auf Styracit.}

Erlenmeyer und Wanklyn') haben, durch Destillation von Mannit und Dulcit mit Jodwasserstoffodure, $\beta$-Hexyljodär $\mathrm{C}_{6} \mathrm{H}_{18} \mathrm{~J}$ (Sdp. $167^{\circ}$ ) dargestellt. Wenn Styracit durch die gleiche Behandlung $\beta$-Hexyljodur lieferte, so könnte man schließen, daß er auch 6 Atome Kohlenstoff in einer Kette enthält.

$15 \mathrm{~g}$ Styracit wurden mit $150 \mathrm{~g}$ Jodwasserstoffsaure (57 \%), unter Zusatz von gelbem Phosphor und unter Durchleiten eines Kohlensăurestromes, destilliert. Sobald im Destillierkolben violetter Joddampf bemerkbar wurde, so wurde die Destillation unterbrochen. Nach dem Erkalten wurden wieder $10 \mathrm{~g}$ Styracit eingetragen, die überdestillierte Jodwasserstoffsăure zurückgegossen and abermals destilliert. Das schwere, dankelgefärbte, ölige Destillat wurde getrennt, durch Schutteln mit Natronlauge enttärbt, durch Calciumchlorid getrocknet und rektifiziert. Es ging bei $165-170^{\circ}$ ein nach Jodamyl riechendes, sich rasch brännendes Oel über. Die Menge des Oels betrug etwa $8 \mathrm{~g}$.

$0,1468 \mathrm{~g}$ frisch destilliertes Oel wurden zur Jodbestimmang in $20 \mathrm{~g}$ Alkohol gelost mit $1 \mathrm{~g}$ Natrium eine Stunde auf dem Wasserbade mit Rúckflubkühler gekocht. Die Lobang wurde in eine Schale gebracht und auf dem Wasserbade rom Alkohol befreit. Der Ruckstand wurde mit Wasser verdünnt and durch Silbernitrat gefallt. Es resultierten $0,1641 \mathrm{~g} \Delta \mathrm{gJ}$.

$$
\begin{gathered}
\text { Berechnet far } \mathrm{C}_{6} \mathrm{H}_{10} \mathrm{~J}: \\
\mathrm{J} \text { : }
\end{gathered}
$$

Aus dem ermittelten Prozentgehalt an Jod und dem Siedepunkt des Oels läßt sich erkennen, daß nichts anderes als $\beta$-Hexyljodïr vorlag.

Weitere Untersuchungen des Styracits, insbesondere des Oxydationsproduktes, sind noch im Gange, and sollen die Resultate spater in dieser Zeitschrift mitgeteilt werden.

Die Samenkerne lieferten bei der Extraktion durch Aether $18,2 \%$ fettes Oel.

Die Konstanten des durch Pressen erhaltenen Oeles sind: Spez. Gew. $0,974\left(15^{\circ}\right)$, S.-Z. 9,00, V.-Z. 180,00, Hübl'sche J.Z. 127,00, Hehner'sche Zahl 91,00.

Toky 0, den 16. Mai 1907.

1) Liebig's Annalen 135, 129. 